This is the post peer-review accepted manuscript of:

A. Elzanaty, A. Giorgetti and M. Chiani, "Syndrome-Based Encoding of Compressible Sources for M2M Communication," IEEE Global Communications Conference (GLOBECOM), Singapore, 2017, pp. 1-6.

The published version is available online at:

https://doi.org/10.1109/GLOCOM.2017.8254840

(C) 2017 IEEE. Personal use of this material is permitted. Permission from IEEE must be obtained for all other uses, in any current or future media, including reprinting/republishing this material for advertising or promotional purposes, creating new collective works, for resale or redistribution to servers or lists, or reuse of any copyrighted component of this work in other works. 


\title{
Syndrome-Based Encoding of Compressible Sources for M2M Communication
}

\author{
Ahmed Elzanaty, Andrea Giorgetti, and Marco Chiani \\ DEI, University of Bologna, ITALY \\ e-mail:\{ahmed.elzanaty, andrea.giorgetti, marco.chiani\}@unibo.it
}

\begin{abstract}
Data originating from many devices and sensors can be modeled as sparse signals. Hence, efficient compression techniques of such data are essential to reduce bandwidth and transmission power, especially for energy constrained devices within machine to machine communication scenarios. This paper provides accurate analysis of the operational distortion-rate function (ODR) for syndrome-based source encoders of noisy sparse sources. We derive the probability density function of error due to both quantization and pre-quantization noise for a type of mixed distributed source comprising Bernoulli and an arbitrary continuous distribution, e.g., Bernoulli-uniform sources. Then, we derive the ODR for two encoding schemes based on the syndromes of Reed-Solomon (RS) and Bose, Chaudhuri, and Hocquenghem $(\mathrm{BCH})$ codes. The presented analysis allows designing a quantizer such that a target average distortion is achieved. As confirmed by numerical results, the closed-form expression for ODR perfectly coincides with the simulation. Also, the performance loss compared to an entropy based encoder is tolerable.
\end{abstract}

\section{INTRODUCTION}

Most of the signals of interest such as image, audio, video, and generated data from Internet of Things (IoT) devices have fewer degrees of freedom compared to their dimension [1]-[3]. Hence, their size can be significantly decreased by exploiting this sparsity structure, i.e., the ability to describe signals with a small set of coefficients compared to their dimension in some domain, e.g., time, frequency, discrete cosine transform, and wavelet. The ultimate goal of source compression is to reduce data rate and power consumption during transmission, so that machine to machine (M2M) communication between energy constrained devices is feasible. Hence, the main challenge is minimizing the number of transmitted bits while preserving the distortion, due to both quantization and pre-quantization noise, below a predefined threshold.

The key objectives considered while designing lossy source encoders for sparse sources are:

1) Finding a practical source encoder which exploits sparsity to minimize the average rate in the presence of noise.

2) Analyzing the theoretical performance of the encoders in terms of the operational distortion-rate function (ODR), which is practically important for optimal bit allocation among sub-bands in wavelet based schemes [4], [5].

This work was supported in part by the European Commission under the EU-METALIC II project within the framework of Erasmus Mundus Action 2 and in part by the European project EuroCPS (grant no. 644090) under the H2020 framework.
For the first objective, we proposed a signal de-noising technique comprising a uniform quantizer and a syndromebased source encoder to reduce the rate [6]. The performance was evaluated only through Monte-Carlo simulation, where it was empirically shown that this scheme is superior to various encoders including compressed sensing [7]-[10].

Regarding the performance analysis, the ODRs of optimal scaler quantizers have been analyzed for uniformly and exponentially distributed sources [11], [12]. An increasing attention has been given to mixed distributed sources (MDSs), adopted for sparse representation of piecewise smooth signals including images in the wavelet domain [13]-[15]. In particular, asymptotic formulations of the information distortion-rate function (IDR) for a class of MDSs have been given in [16], [17]. Moreover, approximate asymptotic expressions for the ODR of uniform quantizers, without considering any saturation effect, have been derived for Bernoulli-Gaussian sources [14], [18].

On the contrary, this paper provides an accurate performance analysis of the proposed lossy source encoder (i.e., uniform quantizer followed by syndrome encoding) for MDSs comprising Bernoulli and an arbitrary continuous distribution with more practical considerations, e.g.,

- Noisy sources with additive pre-quantization noise are analyzed, as the acquisition devices usually induce additional noise. This model fits also the case of not exactly sparse sources (compressible), where the Gaussian noise accounts for the insignificant source symbols [15].

- The considered quantizers have a finite number of levels and they exhibit a saturation effect.

- The achievable syndrome-based encoders are used instead of those based on theoretical entropy rates.

- A closed form expression for the ODR is derived for finite rates, in contrast to the asymptotic analysis for low distortion in the literature.

In particular, we derive the probability density function (PDF) of error due to both quantization and input noise. Then, we find the induced distortion and ODR of two lossy source encoders based on Reed-Solomon (RS) and Bose, Chaudhuri, and Hocquenghem $(\mathrm{BCH})$ syndrome encoding schemes.

Throughout the paper, small letters denote scalars and random variable (r.v.) realizations, bold letters denote vectors, calligraphic letters indicate r.v.s, $\mathcal{N}(\boldsymbol{\mu}, \mathbf{C})$ denotes the multivariate Gaussian distribution with mean $\boldsymbol{\mu}$ and covariance matrix $\mathbf{C}, \mathbf{I}_{N}$ is the $N$-dimensional identity matrix, $\mathbb{F}_{q}$ denotes 


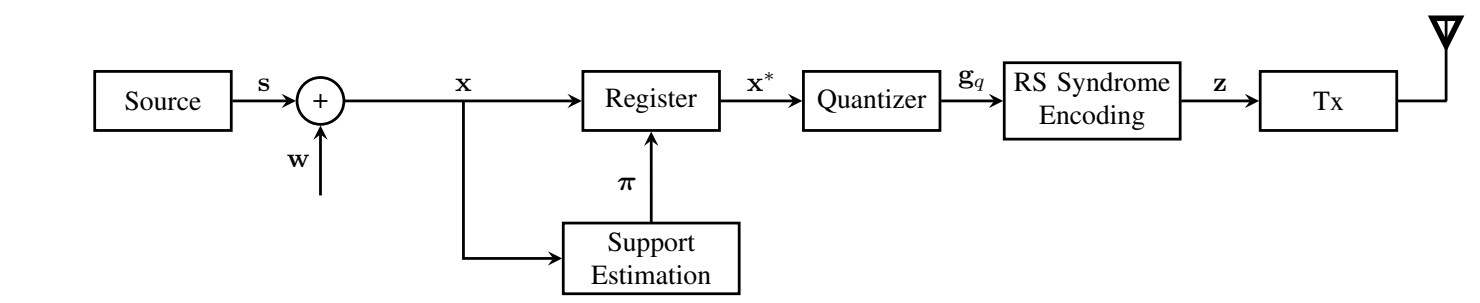

(a) RS based source coding (RSSC).

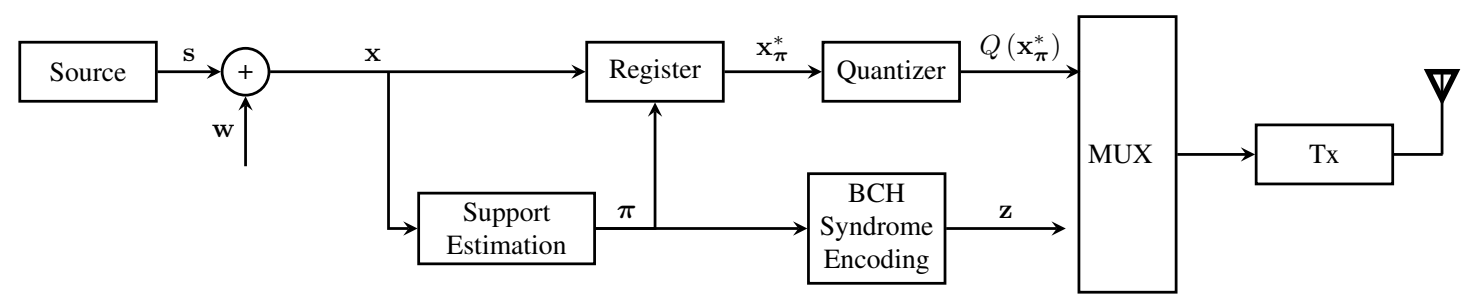

(b) $\mathrm{BCH}$ based source coding (BCHSC).

Figure 1. The block diagram of the proposed compression schemes for noisy sparse sources.

the Galois field of order $q$, and $\lfloor$.$\rfloor and \lceil$.$\rceil represent the$ flooring and ceiling functions, respectively.

\section{SignAl MODEL}

The source emits independent, identically distributed (i.i.d.) r.v.s collected into a vector of length $N, \mathbf{s} \in \mathbb{R}^{N}$. Each source symbol, $\mathcal{S}$, is generated as a multiplication of a Bernoulli r.v. $\mathcal{Z}$ (with probability $p$ of generating ones) and $\mathcal{Y}$ drawn from some continuous distribution $P_{\mathcal{Y}}(y)$. Hence, the corresponding $\mathrm{PDF}$ of $\mathcal{S}$ is

$$
P_{\mathcal{S}}(s)=p P_{\mathcal{Y}}(s)+(1-p) \delta(s)
$$

The acquisition device may induce some noise to the input signal, thus the noisy source vector can be represented as

$$
\mathbf{x}=\mathbf{s}+\mathbf{w}
$$

where $\mathbf{w} \sim \mathcal{N}\left(\mathbf{0}, \sigma_{\mathrm{n}}^{2} \mathbf{I}_{N}\right)$ represents the additive noise.

\section{THE PRoposed COMPRESSION SCHEMES BASED ON SYNDROME ENCODING}

In this section, we briefly shed the light on two schemes for lossy source compression proposed in [6] (see Fig. 1). Let us consider a random realization of the noiseless source, $s$, with $k_{0}$ non-zero elements in locations identified by the ones in $\pi \in\{0,1\}^{N}$. At first, the locations of the non-zero elements should be estimated through a support estimation mechanism, e.g., power detection and model order selection [6], [19], [20]. Assuming perfect support estimation, the encoder nulls out the $N-k_{0}$ noise components outside the non-zero elements support as

$$
x_{i}^{*}=\left\{\begin{array}{lr}
x_{i}, & i \in \boldsymbol{\pi} \\
0, & \text { otherwise }
\end{array}\right.
$$

\section{A. Scalar Uniform Quantizer}

Due to the large number of zero elements in $\mathrm{x}^{*}$, we consider a scalar mid-tread uniform quantizer, whose zerovalued reconstruction level prevents the introduction of additional quantization noise out of the signal support [21]. This quantizer maps each element $x_{i}^{*}$ of $\mathrm{x}^{*}$ to a discrete quantization index

$$
Q: \mathbb{R} \rightarrow\left\{0,1, \cdots, 2^{b}-2\right\}
$$

where $b$ is the quantization depth indicating the number of bits per sample. More precisely, the signal $x^{*}$ is uniformly partitioned into $2^{b}-1$ levels with a step size

$$
\Delta=\frac{2 A}{2^{b}-1}
$$

where $[-A, A]$ is the supported range beyond which the output is saturated. The index vector of the quantized signal is then

$$
\mathbf{g}_{q} \triangleq Q\left(\mathbf{x}^{*}\right)=\left(Q\left(x_{1}^{*}\right), Q\left(x_{2}^{*}\right), \cdots, Q\left(x_{N}^{*}\right)\right) \in \mathbb{F}_{q}^{N} .
$$

\section{B. Syndrome-Based Source Encoder}

Regarding the RS based source coding (RSSC) approach, the quantized signal $\mathbf{g}_{q}$ is compressed by calculating its syndrome vector $\mathbf{z}$ through the parity check matrix of the $k_{0^{-}}$ error-correcting RS code at the encoder depicted in Fig.1(a). The resulting total number of bits required to encode the sparse vector using RS syndrome coding is

$$
R_{\mathrm{RS}}=2 k_{0} b=2 k_{0} \log _{2}(N+1)
$$

where $b \geq 3, N=2^{b}-1$. The locations and values of the non-zero elements can be estimated at the receiver from the syndrome vector $\mathbf{z}$, which is the sparsest vector satisfying the computed syndrome, using Berlekamp's algorithm [22]. This is attributed to the duality between source and channel coding problems, where the maximum sparsity order is consistent with the correcting capability of the chosen channel code. 
A further compression gain can be achieved by separately sending the $k_{0}$ quantized non-zero elements, then compressing the binary vector which determines their locations using the syndrome of a $\mathrm{BCH}$ code, as shown in Fig.1(b). In fact, the required number of bits for the source encoding is

$$
R_{\mathrm{BCH}}=m\left(N, k_{0}\right)+k_{0} b \leq k_{0} \log _{2}(N+1)+k_{0} b
$$

where $m\left(N, k_{0}\right) \leq k_{0} \log _{2}(N+1)$ is the number of parity check bits calculated from the design table of the BCH code, for a given sparsity order $k_{0}$ (i.e., error correcting capability) and dimension $N$ [23, Appendix C]. Clearly, from (5) and (6), the rate is upper-bounded by the RS based approach. In the decoder, the binary location vector can also be recovered using the Berlekamp's algorithm, and the quantized non-zero entries are reconstructed from the quantization indexes as usual.

\section{Operational Distortion-Rate Analysis}

In this section, we derive the ODR for the proposed schemes in Fig. 1, which is also considered as an upper bound on the IDR for sparse memoryless sources. The ODR, $D(R)$, is a function that maps the rate $R$ at which the system is working (i.e., the average number of bits required to describe a single source sample) to the mean square error distortion due to both quantization and pre-quantization noise. More precisely,

$$
D=\mathbb{E}\left[(\hat{\mathcal{S}}(\mathcal{S}, \mathcal{W}, R)-\mathcal{S})^{2}\right]=\int_{-\infty}^{\infty} \tau^{2} P_{\mathcal{T}}(\tau) d \tau
$$

where $\mathcal{S} \sim P_{\mathcal{S}}(s), \mathcal{W} \sim \mathcal{N}\left(0, \sigma_{\mathrm{n}}^{2}\right), \hat{\mathcal{S}}$, and $\mathcal{T} \triangleq \hat{\mathcal{S}}-\mathcal{S}$ are r.v.s representing the source output, pre-quantization noise, decoder output, and error due to both the quantization and pre-quantization noise, respectively. ${ }^{1}$ Hence, finding the PDF of error, $P_{\mathcal{T}}(\tau)$, is essential for deriving the distortion-rate function. More precisely, the decoder output can be written, after off-support de-noising in (3), as

$$
\begin{aligned}
\hat{\mathcal{S}} & =Q^{-1}\left(Q\left(\mathcal{X}^{*}\right)\right)=Q^{-1}(Q(\mathcal{S}+\mathcal{Z W})) \\
& =Q^{-1}(Q(\mathcal{Z} \mathcal{Y}+\mathcal{Z W})) \triangleq \mathcal{S}+\mathcal{T}
\end{aligned}
$$

where $\mathcal{X}^{*}$ is the r.v. representing the filtered signal in (3) and $Q^{-1}(Q(x))$ is the scalar mid-tread uniform quantizer with bounded range which is defined as

$Q^{-1}(Q(x))=\left\{\begin{array}{lc}-A+\Delta / 2, & x \leq-A+\Delta \\ i \Delta, & \left(i-\frac{1}{2}\right) \Delta \leq x \leq\left(i+\frac{1}{2}\right) \Delta \\ A-\Delta / 2, & x \geq A-\Delta\end{array}\right.$

with $i \in\left\{i_{\min }+1, i_{\min }+2, \ldots, i_{\max }-1\right\}$, where $i_{\max }=$ $-i_{\min }=\left(2^{b}-2\right) / 2=A / \Delta-1 / 2$. Clearly, the PDF of the error $\mathcal{T}$ can be written as

$$
\begin{aligned}
P_{\mathcal{T}}(\tau) & =\sum_{j=1}^{2} P_{\mathcal{T} \mid \mathcal{Z}}\left(\tau \mid z_{j}\right) P_{\mathcal{Z}}\left(z_{j}\right) \\
& =p P_{\mathcal{T} \mid \mathcal{Z}}(\tau \mid 1)+(1-p) P_{\mathcal{T} \mid \mathcal{Z}}(s \mid 0) \\
& =p P_{\mathcal{T} \mid \mathcal{Z}}(\tau \mid 1)+(1-p) \delta(\tau)
\end{aligned}
$$

\footnotetext{
${ }^{1}$ Since the source is memoryless, scalars rather than vectors are considered and the subscript $i$ is dropped from the notation.
}

where the error vanishes for $z=0$, as the support is perfectly estimated at the encoder and mid-tread quantizers do not introduce distortion to the zero valued source symbol. Hence, only the non-zero entities are subjected to distortion. The remaining part is the PDF of error given that the source symbol is non-zero, which can be written as

$$
\begin{aligned}
P_{\mathcal{T} \mid \mathcal{Z}}(\tau \mid 1)= & \sum_{i=i_{\min }}^{i_{\max }} P_{\hat{\mathcal{S}}, \mathcal{Y} \mid \mathcal{Z}}\left(\hat{s}_{i}, \hat{s}_{i}-\tau \mid 1\right) \\
= & P_{\hat{\mathcal{S}} \mid \mathcal{Y}, \mathcal{Z}}\left(\hat{s}_{i_{\min }} \mid \hat{s}_{i_{\min }}-\tau, 1\right) P_{\mathcal{Y}}\left(\hat{s}_{i_{\min }}-\tau\right) \\
& +\sum_{i=i_{\min }+1}^{i_{\max }-1} P_{\hat{\mathcal{S}} \mid \mathcal{Y}, \mathcal{Z}}\left(\hat{s}_{i} \mid \hat{s}_{i}-\tau, 1\right) P_{\mathcal{Y}}\left(\hat{s}_{i}-\tau\right) \\
& +P_{\hat{\mathcal{S}} \mid \mathcal{Y}, \mathcal{Z}}\left(\hat{s}_{i_{\max }} \mid \hat{s}_{i_{\max }}-\tau, 1\right) P_{\mathcal{Y}}\left(\hat{s}_{i_{\max }}-\tau\right) .
\end{aligned}
$$

The probability that the decoder selects $\hat{s}_{i_{\min }}$ given that the entry is non-zero and has the value $\hat{s}_{i_{\min }}-\tau$ equals the probability that noise is bounded below $\tau+\Delta / 2$, i.e.,

$$
\begin{aligned}
P_{\hat{\mathcal{S}} \mid \mathcal{Y}, \mathcal{Z}} & \left(\hat{s}_{i_{\min }} \mid \hat{s}_{i_{\min }}-\tau, 1\right)=\mathbb{P}\left\{\hat{s}_{i_{\min }}-\tau+\mathcal{W} \leq-A+\Delta\right\} \\
& =\mathbb{P}\{-A+\Delta / 2-\tau+\mathcal{W} \leq-A+\Delta\} \\
& =\mathbb{P}\left\{\mathcal{W} \leq \tau+\frac{\Delta}{2}\right\}=\phi(\tau)
\end{aligned}
$$

where $\phi(x)=\left[1+\operatorname{erf}\left((x+\Delta / 2) /\left(\sqrt{2} \sigma_{\mathrm{n}}\right)\right)\right] / 2$ and $\hat{s}_{i_{\min }}$ is obtained from (9). Similarly,

$$
\begin{aligned}
P_{\hat{\mathcal{S}} \mid \mathcal{Y}, \mathcal{Z}} & \left(\hat{s}_{i_{\max }} \mid \hat{s}_{i_{\max }}-\tau, 1\right)=\mathbb{P}\left\{\hat{s}_{i_{\max }}-\tau+\mathcal{W} \geq A-\Delta\right\} \\
& =\mathbb{P}\{A-\Delta / 2-\tau+\mathcal{W} \geq A-\Delta\} \\
& =\mathbb{P}\left\{\mathcal{W} \geq \tau-\frac{\Delta}{2}\right\}=\phi(-\tau) .
\end{aligned}
$$

The probability to have $s$ in the output with $i \in\left\{i_{\min +1}\right.$ : $\left.i_{\max -1}\right\}$ given $\mathcal{Y}$ and that the source symbol is non-zero can be written as

$$
\begin{aligned}
& P_{\hat{\mathcal{S}} \mid \mathcal{Y}, \mathcal{Z}}\left(\hat{s}_{i} \mid \hat{s}_{i}-s, 1\right) \\
& =\mathbb{P}\left\{i \Delta-\Delta / 2-\hat{s}_{i}+\tau \geq \mathcal{W} \geq-\hat{s}_{i}+\tau+i \Delta+\Delta / 2\right\} \\
& =\mathbb{P}\{\tau-\Delta / 2 \geq \mathcal{W} \geq \tau+\Delta / 2\}=\Phi(\tau)
\end{aligned}
$$

where $\hat{s}_{i}=i \Delta$ and

$$
\Phi(x) \triangleq \frac{1}{2}\left[\operatorname{erf}\left(\frac{x+\Delta / 2}{\sqrt{2} \sigma_{\mathrm{n}}}\right)-\operatorname{erf}\left(\frac{x-\Delta / 2}{\sqrt{2} \sigma_{\mathrm{n}}}\right)\right] .
$$

Altogether, (10)-(14) give the error density function for an arbitrary distribution of the non-zero elements $P_{\mathcal{Y}}(y)$ as

$$
\begin{gathered}
P_{\mathcal{T}}(\tau)=(1-p) \delta(\tau)+p(\underbrace{\Phi(\tau) \sum_{i=-A / \Delta+3 / 2}^{A / \Delta-3 / 2} P_{\mathcal{Y}}\left(\hat{s}_{i}-\tau\right)}_{P_{3}} \\
+\underbrace{\phi(\tau) P_{\mathcal{Y}}\left(\hat{s}_{i_{\min }}-\tau\right)}_{P_{1}}+\underbrace{\phi(-\tau) P_{\mathcal{Y}}\left(\hat{s}_{i_{\max }}-\tau\right)}_{P_{2}}) .
\end{gathered}
$$


Let us consider Bernoulli-uniform (BU) sources, where the non-zero elements are drawn from a uniform distribution with

$$
P_{\mathcal{Y}}(y)= \begin{cases}\frac{1}{2 A}, & |y| \leq A \\ 0, & \text { otherwise. }\end{cases}
$$

The most important consideration while substituting (16) into $(15)$ is to find the proper intervals adherent with r.v. $\mathcal{Y}$ support. For ease of clarity, each part of (15) will be treated separately. In particular, $P_{1}$ and $P_{2}$ can be found such that $\left|\hat{s}_{i_{\min }}-\tau\right| \leq A$ and $\left|\hat{s}_{i_{\max }}-\tau\right| \leq A$, respectively, as

$$
\begin{aligned}
& P_{1}=\frac{1}{2 A}\left\{\begin{array}{lr}
\phi(\tau), & -2 A+\frac{\Delta}{2} \leq \tau \leq \frac{\Delta}{2} \\
0, & \text { otherwise }
\end{array}\right. \\
& P_{2}=\frac{1}{2 A}\left\{\begin{array}{lr}
\phi(-\tau), & -\frac{\Delta}{2} \leq \tau \leq 2 A-\frac{\Delta}{2} \\
0, & \text { otherwise. }
\end{array}\right.
\end{aligned}
$$

Regarding $P_{3}$, two conditions should be satisfied while finding the range of $\tau$ such that $P_{3}$ is non-zero, i.e., $\left|\hat{s}_{i}-\tau\right| \leq A$ leading to

$$
\begin{aligned}
\tau-A & \leq \hat{s}_{i} \leq A+\tau \\
\lceil(\tau-A) / \Delta\rceil & \leq i \leq\lfloor(A+\tau) / \Delta\rfloor
\end{aligned}
$$

and the second constraint from the summation limits in (15)

$$
-A / \Delta+3 / 2 \leq i \leq A / \Delta-3 / 2 .
$$

By considering the inequalities of $i$, we get

$$
P_{\mathcal{Y}}\left(\hat{s}_{i}-\tau\right)=\frac{1}{2 A}\left\{\begin{array}{lr}
1, & -2 A+\frac{3}{2} \Delta \leq \tau \leq 2 A-\frac{3}{2} \Delta \\
0, & \text { otherwise }
\end{array}\right.
$$

with $i: i_{L}(\tau) \leq i \leq i_{H}(\tau)$, where

$$
\begin{aligned}
& i_{L} \triangleq \max \{\lceil(\tau-A) / \Delta\rceil,-A / \Delta+3 / 2\} \\
& i_{H} \triangleq \min \{\lfloor(A+\tau) / \Delta\rfloor, A / \Delta-3 / 2\} .
\end{aligned}
$$

Since the distribution is uniform, the summation in (15) reduces to $\left(i_{H}(\tau)-i_{L}(\tau)+1\right) / 2 A$. It has three distinct values depending on $\tau$ range, therefore $P_{3}$ can be represented as

$$
P 3=\frac{1}{2 A}\left\{\begin{array}{c}
(\lfloor(A+\tau) / \Delta\rfloor+A / \Delta-0.5) \Phi(\tau), \\
\text { if }-2 A+1.5 \Delta \leq \tau<-1.5 \Delta \\
(2 A / \Delta-2) \Phi(\tau), \\
\quad \text { if }-1.5 \Delta \leq \tau<1.5 \Delta \\
(-\lceil(\tau-A) / \Delta\rceil+A / \Delta-0.5) \Phi(\tau), \\
\quad \text { if } 1.5 \Delta \leq \tau<2 A-1.5 \Delta \\
0, \quad \text { otherwise. }
\end{array}\right.
$$

From (15) to (19), the PDF of pre-quantization plus quantization noise error can be written as

$$
\begin{aligned}
& P_{\mathcal{T}}(\tau)=(1-p) \delta(\tau)+ \\
& \frac{p}{2 A}\left\{\begin{array}{c}
(2 A / \Delta-1) \Phi(\tau), \\
(2 A / \Delta-2) \Phi(\tau)+\phi(-\tau), \\
\text { if } \Delta / 2 \leq|\tau|<3 \Delta / 2 \\
\Phi(\tau)(A / \Delta+\lfloor(A-\tau) / \Delta\rfloor-1 / 2)+\phi(-\tau), \\
\text { if } 3 \Delta / 2 \leq|\tau|<2 A-3 \Delta / 2 \\
\phi(-\tau), \quad \text { if } 2 A-3 \Delta / 2 \leq|\tau|<2 A-\Delta / 2 .
\end{array}\right.
\end{aligned}
$$

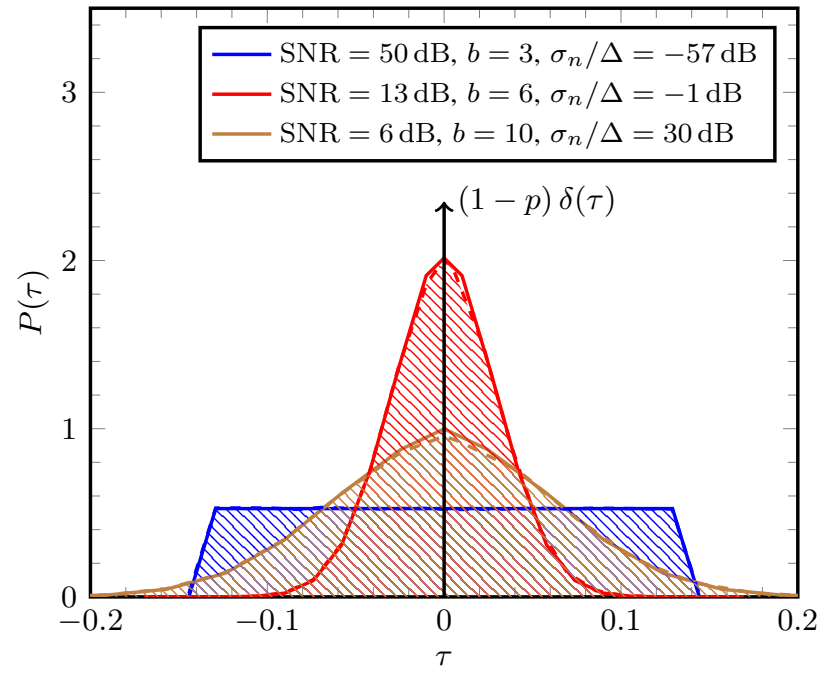

Figure 2. The PDF of error both analytical (solid) and empirical (dashed with hashed area), for various SNRs and bit depths $b$.

Regarding the induced distortion at the decoder output, from (7) and (20) we have

$$
\begin{aligned}
D & \simeq \widehat{D}(\Delta) \triangleq \int_{-\infty}^{\infty} \tau^{2} \tilde{P}_{\mathcal{T}}(\tau) d \tau \\
& \simeq p\left(\frac{\Delta^{2}}{12}+\sigma_{\mathrm{n}}^{2}\right)=p\left(\frac{A^{2}}{3\left(2^{b}-1\right)^{2}}+\sigma_{\mathrm{n}}^{2}\right)
\end{aligned}
$$

where $\tilde{P}_{\mathcal{T}}(\tau)$ is an accurate approximation of the PDF in (20) by considering that $\lfloor x\rfloor \simeq x-1 / 2$. The integral (21) is exactly calculated and reported in (27) at the bottom of the last page.

Now, the ODR for RSSC in Fig. 1(a) can be derived by reformulating the distortion in terms of the average rate. At first, the system expected rate is calculated as

$$
R=\mathbb{E}\left[R_{\mathrm{RS}} / N\right]=2 b_{\mathrm{RS}} \mathbb{E}\left[\mathcal{K}_{0} / N\right]=2 b_{\mathrm{RS}} p
$$

where $\mathcal{K}_{0}$ is a r.v. distributed as a binomial distribution with mean $p$ and it represents the number of non-zero elements. Hence, the ODR can be written from (4), (22), and (23) as

$$
D_{\mathrm{RS}}(R) \simeq \widehat{D}\left(\frac{2 A}{2^{b_{\mathrm{RS}}}-1}\right) \simeq p\left(\frac{A^{2}}{3}\left(2^{R / 2 p}-1\right)^{-2}+\sigma_{\mathrm{n}}^{2}\right) .
$$

Similarly, the expected rate for $\mathrm{BCH}$ based encoder is

$$
\begin{aligned}
R & =\mathbb{E}\left[R_{\mathrm{BCH}} / N\right]=\mathbb{E}\left[\left(b_{\mathrm{BCH}} \mathcal{K}_{0}+m\left(N, \mathcal{K}_{0}\right)\right) / N\right] \\
& =p b_{\text {всн }}+m(N, p N) / N \leq p\left(b_{\mathrm{BCH}}+\log _{2}(N+1)\right) .
\end{aligned}
$$

Finally, the ODR of the $\mathrm{BCH}$ based scheme is found as

$$
\begin{aligned}
D_{\mathrm{BCH}}(R) & \simeq \widehat{D}\left(\frac{2 A}{2^{b_{\mathrm{BCH}}}-1}\right) \\
& \simeq p\left(\frac{A^{2}}{3}\left(2^{R / p-m(N, p N) /(p N)}-1\right)^{-2}+\sigma_{\mathrm{n}}^{2}\right) \\
& \leq p\left(\frac{A^{2}}{3}\left(2^{R / p-\log _{2}(N+1)}-1\right)^{-2}+\sigma_{\mathrm{n}}^{2}\right) .
\end{aligned}
$$




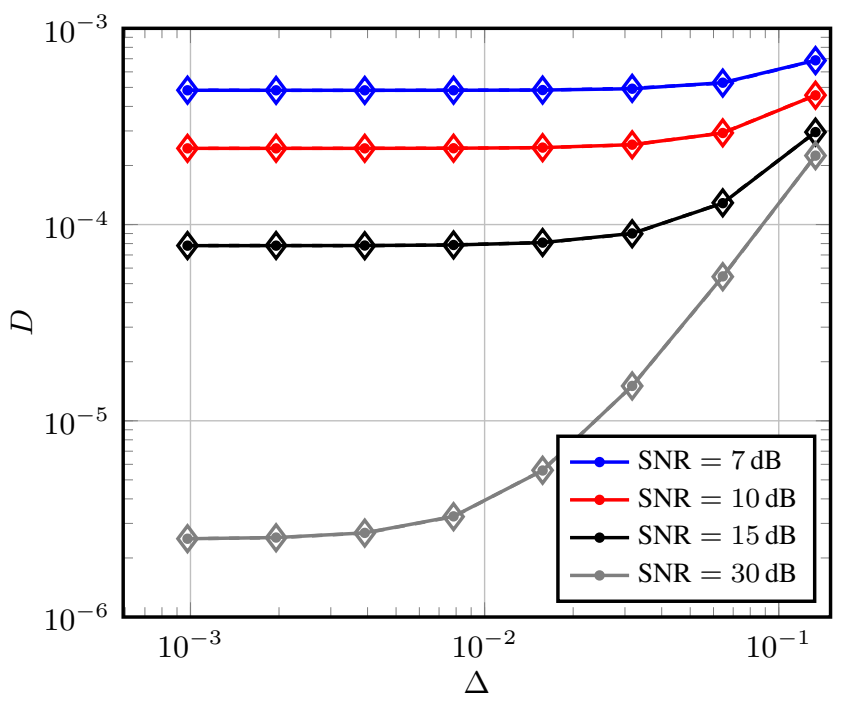

Figure 3. The MSE vs step size, for various SNRs. The analytical performance $(\operatorname{solid} *)$ perfectly coincides with the simulation (dashed $\diamond)$.

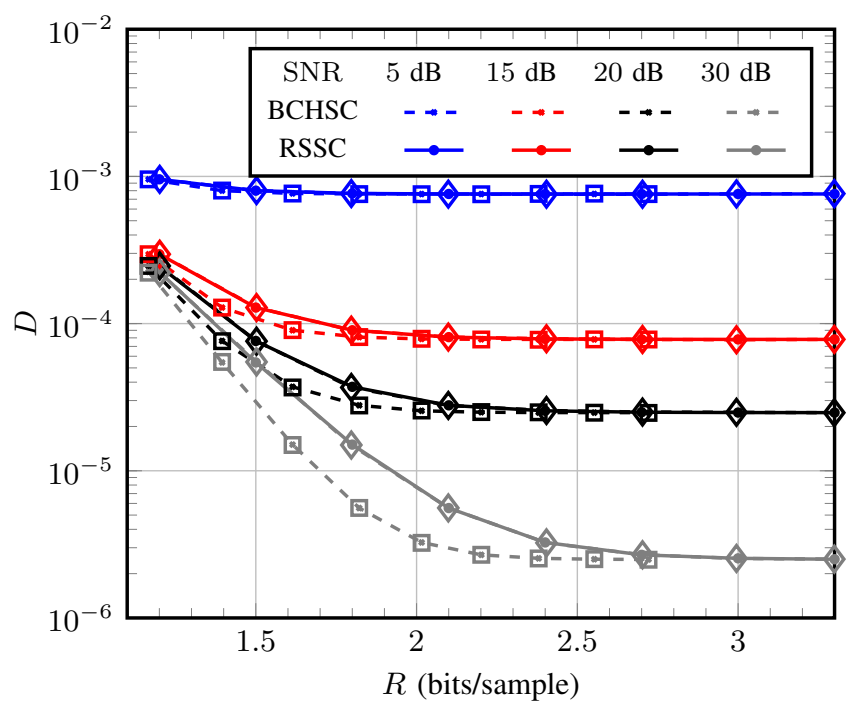

Figure 4. The ODR for the proposed BCHSC (solid) and RSSC (dashed) schemes, for $b \in\{4,5, \ldots, 12\}$ and $\mathrm{SNR} \in\{5,15,20,30\} \mathrm{dB}$.

\section{NumericAl Results}

In this section, numerical results and Monte-Carlo simulations are presented to illustrate the performance of the proposed RSSC and BCHSC schemes. We also compare the ODR of such encoders with an entropy-based bound. The signal-to-noise ratio (SNR) is the signal power to the prequantization noise power at the encoder which is defined as $\mathrm{SNR} \triangleq 10 \log _{10}\left(\mathbb{E}\left\{\mathbf{s} \mathbf{s}^{T}\right\} / \mathbb{E}\left\{\mathbf{w} \mathbf{w}^{T}\right\}\right) \mathrm{dB}$. In all numerical results $p=0.15, A=1$, and $N=2^{b}-1$, respectively.

In Fig. 2, we show the PDF of error due to both quantization and pre-quantization noise for $\mathrm{SNR} \in\{50,13,6\} \mathrm{dB}$ and $b \in\{3,6,10\}$ bits per sample. As we can see the theoretical PDF in (20) agrees with the normalized histogram obtained from simulation. Note also that the PDF is a mixture

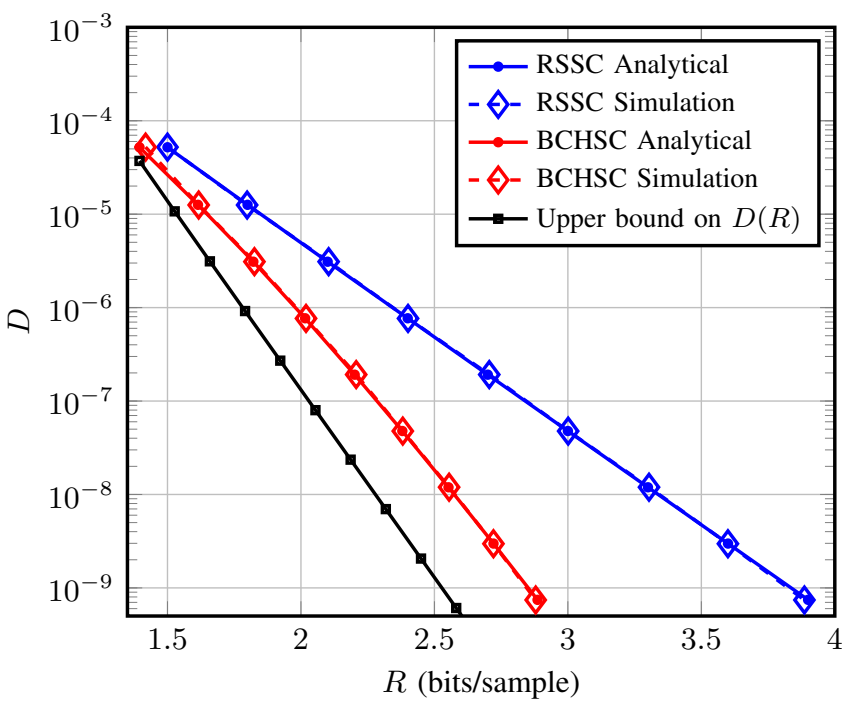

Figure 5. The ODR of BCHSC and RSSC along with an upper bound on the IDR, with $b \in\{5, \ldots, 13\}$ and $\mathrm{SNR}=\infty$.

distribution with a Dirac delta function at zero with weight $1-p$ representing a perfect recovery of the zero elements at the decoder. On the other hand, the PDF of the non-zero entries is a combination of error due to quantization only (uniform distribution from $-\Delta / 2$ to $\Delta / 2$ ) and distortion from the pre-quantization noise (zero mean Gaussian distribution with variance $\sigma_{\mathrm{n}}^{2}$ ). The resulting shape depends on $\sigma_{\mathrm{n}} / \Delta$, e.g., $P(\tau)$ tends more to uniform distribution for small ratios.

Fig. 3 shows the MSE distortion (27) vs step size $\Delta$ of the uniform quantizer with $b \in\{4,5, \ldots, 12\}$, for noisy BU sources with $\mathrm{SNR} \in\{7,10,15,30\} \mathrm{dB}$. The distortion increases with both the step size and noise variance. Moreover, the minimum distortion that one can achieve depends on the amount of prequantization noise, e.g, decreasing $\Delta$ from $10^{-2}$ to $10^{-3}$ has negligible effect on the distortion for $\mathrm{SNR} \leq 15 \mathrm{~dB}$.

Fig. 4 illustrates the operational distortion-rate function of RSSC (24) (dashed) and BCHSC (26) (solid) schemes with $b \in\{4,5, \ldots, 12\}$ and $\mathrm{SNR} \in\{5,15,20,30\} \mathrm{dB}$. Note the analytical performance for (RSSC $*$ ) and (BCHSC •) perfectly coincides with simulation $(\diamond, \square)$. It is evident that $D_{\mathrm{BCH}}(R) \leq D_{\mathrm{RS}}(R)$ with quite differences at high SNRs which is attributed to $R_{\mathrm{BCH}} \leq R_{\mathrm{RS}}$ from (5) and (6). Also, the distortion tends approximately to $p \sigma_{\mathrm{n}}^{2}$ as $\Delta \rightarrow 0$, for instance, increasing the rate from 2 to 3 bits/sample does not significantly decrease the distortion for low SNRs.

We presents in Fig. 5 upper bounds on the information distortion-rate function through several source compression schemes noting that any achievable ODR provides an upper bound on IDR. In particular, we compare the BCHSC and RSSC with an entropy based encoder, where the location vector is coded with a minimal number of bits indicated by the Bernoulli source entropy and the non-zero values are encoded using a uniform quantizer. More precisely, its average rate is $R=-p \log _{2}(p)-(1-p) \log _{2}(1-p)+p b_{\text {entropy }}$ with ODR 
$\widehat{D}\left(2 A /\left(2^{b_{\text {entropy }}}-1\right)\right)$. We can see that the gap between the BCHSC and entropy based scheme is small, especially in the region of low-rate high-distortion.

\section{CONCLUSION}

This paper provided the distortion rate analysis of two novel schemes for efficient encoding of noisy sparse sources, i.e., Bernoulli-uniform sources. These schemes are particularly important for M2M communication scenarios involving the exchange of sparse signals, e.g, images and data from wearable sensors, to reduce both the consumed power and bandwidth. The analytical performance of the proposed methods perfectly coincides with the simulation results. Additionally, we point out that the ODR of BCHSC is better than that of RSSC. As illustrated by numerical results, the gap between an entropy based source encoder and the proposed approaches is small.

\section{REFERENCES}

[1] M. Vetterli, "Wavelets, approximation, and compression," IEEE Signal Process. Mag., vol. 18, no. 5, pp. 59-73, Sep. 2001.

[2] J. Haupt, W. U. Bajwa, M. Rabbat, and R. Nowak, "Compressed sensing for networked data," IEEE Signal Process. Mag., vol. 25, no. 2, pp. 92101, Mar. 2008

[3] S. Mallat, A wavelet tour of signal processing: the sparse way. Academic press, 2008.

[4] P. H. Westerink, J. Biemond, and D. E. Boekee, "An optimal bit allocation algorithm for sub-band coding," in Proc. IEEE Int. Conf. Acoust. Speech Sig. Proc. (ICASSP), vol. 2, Apr. 1988, pp. 757-760.

[5] A. Ortega and K. Ramn, "Rate-distortion methods for image and video compression," IEEE Signal Process. Mag., vol. 15, no. 6, pp. 23-50, Nov. 1998.

[6] A. Elzanaty, A. Giorgetti, and M. Chiani, "Efficient compression of noisy sparse sources based on syndrome encoding," in Proc. IEEE Int. Conf. on Global Comm. (GLOBECOM), Washington DC, USA, Dec. 2016, pp. 1-6.

[7] E. Candes and T. Tao, "Decoding by linear programming," IEEE Trans. Inf. Theory, vol. 51, no. 12, pp. 4203-4215, Dec. 2005.

[8] F. Chen, A. P. Chandrakasan, and V. M. Stojanovic, "Design and analysis of a hardware-efficient compressed sensing architecture for data compression in wireless sensors," IEEE J. Solid-State Circuits, vol. 47, no. 3, pp. 744-756, Mar. 2012.

[9] M. Chiani, A. Elzanaty, and A. Giorgetti, "Analysis of the restricted isometry property for Gaussian random matrices," in Proc. IEEE Int. Conf. on Global Comm. (GLOBECOM), San Diego, USA, Dec. 2015, pp. $1-6$.
[10] A. Elzanaty, A. Giorgetti, and M. Chiani, "On sparse recovery using finite Gaussian matrices: RIP-based analysis," in Proc. IEEE Stati. Signal Processing Work. (SSP), Palma de Mallorca, Spain, Jun. 2016, pp. 395399.

[11] A. Gyorgy and T. Linder, "Optimal entropy-constrained scalar quantization of a uniform source," IEEE Trans. Inf. Theory, vol. 46, no. 7, pp. 2704-2711, Nov. 2000.

[12] G. J. Sullivan, "Efficient scalar quantization of exponential and laplacian random variables," IEEE Trans. Inf. Theory, vol. 42, no. 5, pp. 13651374, Sep. 1996.

[13] D. Leporini, J.-C. Pesquet, and H. Krim, Best Basis Representations with Prior Statistical Models. New York, NY: Springer New York, 1999, pp. $155-172$.

[14] A. Fraysse, B. Pesquet-Popescu, and J. C. Pesquet, "On the uniform quantization of a class of sparse sources," IEEE Trans. Inf. Theory, vol. 55, no. 7, pp. 3243-3263, July 2009.

[15] C. Weidmann and M. Vetterli, "Rate distortion behavior of sparse sources," IEEE Trans. Inf. Theory, vol. 58, no. 8, pp. 4969-4992, Aug. 2012.

[16] H. Rosenthal and J. Binia, "On the epsilon entropy of mixed random variables," IEEE Trans. Inf. Theory, vol. 34, no. 5, pp. 1110-1114, Sep. 1988.

[17] A. Gyorgy, T. Linder, and K. Zeger, "On the rate-distortion function of random vectors and stationary sources with mixed distributions," IEEE Trans. Inf. Theory, vol. 45, no. 6, pp. 2110-2115, Sep. 1999.

[18] M. Kaaniche, A. Fraysse, B. Pesquet-Popescu, and J. C. Pesquet, "Accurate rate-distortion approximation for sparse bernoulli-generalized gaussian models," in Proc. IEEE Int. Conf. Acoust. Speech. Signal. Process. (ICASSP), Florence, Italy, May 2014, pp. 2020-2024.

[19] A. Mariani, A. Giorgetti, and M. Chiani, "Model order selection based on information theoretic criteria: Design of the penalty," IEEE Trans. Signal Process., vol. 63, no. 11, pp. 2779-2789, Jun. 2015.

[20] - "Wideband spectrum sensing by model order selection," IEEE Trans. Wireless Commun., vol. 14, no. 12, pp. 6710-6721, Dec. 2015.

[21] Y. You, Audio Coding: Theory and Applications. Springer Science \& Business Media, 2010.

[22] E. R. Berlekamp, Algebraic coding theory, ser. McGraw-Hill series in systems science. New York, NY: McGraw-Hill, 1968.

[23] J. Daniel and J. Costello, Error control coding, Fundamentals and applications. Prentice Hall, Inc. Englewood Cliffs, New Jersey, 1983.

$$
\begin{aligned}
& \widehat{D}(\Delta)=p\left(\frac{\Delta^{2}}{12}+\sigma^{2}+\frac{1}{48 \sqrt{\pi} A \Delta}\left[e ^ { - \frac { 2 ( A ^ { 2 } + \Delta ^ { 2 } ) } { \sigma ^ { 2 } } } \left(\sigma \left(-16 \sqrt{2} A^{3}\left(e^{\frac{4 A \Delta}{\sigma^{2}}}-e^{\frac{\Delta(4 A+3 \Delta)}{2 \sigma^{2}}}\right)-\right.\right.\right.\right. \\
& \Delta\left(\sqrt{2} e^{\frac{2 A^{2}}{\sigma^{2}}}\left(4 \sigma^{2}-\Delta^{2}\right)+2 \sqrt{2}\left(\Delta^{2}+5 \sigma^{2}\right) e^{\frac{4 A^{2}+3 \Delta^{2}}{2 \sigma^{2}}}+24 \sqrt{\pi} \Delta \sigma e^{\frac{2\left(A^{2}+\Delta^{2}\right)}{\sigma^{2}}}-2 \sqrt{2}\left(\Delta^{2}+5 \sigma^{2}\right) e^{\frac{\Delta(4 A+3 \Delta)}{2 \sigma^{2}}+}\right. \\
& \left.\sqrt{2}\left(\Delta^{2}-4 \sigma^{2}\right) e^{\frac{4 A \Delta}{\sigma^{2}}}\right)+8 \sqrt{2} A^{2} \Delta\left(2 e^{\frac{4 A \Delta}{\sigma^{2}}}-7 e^{\frac{\Delta(4 A+3 \Delta)}{2 \sigma^{2}}}\right)-2 \sqrt{2} A\left(\left(2 \sigma^{2}-7 \Delta^{2}\right) e^{\frac{\Delta(4 A+3 \Delta)}{2 \sigma^{2}}}+\right. \\
& \left.\left.\left(\Delta^{2}-2 \sigma^{2}\right) e^{\frac{4 A \Delta}{\sigma^{2}}}\right)\right)+\sqrt{\pi} e^{\frac{2\left(A^{2}+\Delta^{2}\right)}{\sigma^{2}}}\left(-\left(2(2 A-\Delta)\left(8 A^{3}-28 A^{2} \Delta+7 A \Delta^{2}+\Delta^{3}\right)+3 \Delta \sigma^{2}(8 A+3 \Delta)\right.\right. \\
& \left.+18 \sigma^{4}\right) \operatorname{erfc}\left(\frac{2 A-\Delta}{\sqrt{2} \sigma}\right)+\left(32 A^{4}-64 A^{3} \Delta+36 A^{2} \Delta^{2}-2 A\left(\Delta^{3}+12 \Delta \sigma^{2}\right)-2 \Delta^{4}+21 \Delta^{2} \sigma^{2}+18 \sigma^{4}\right) \\
& \left.\left.\left.\left.\operatorname{erfc}\left(\frac{\sqrt{2}(A-\Delta)}{\sigma}\right)+\left(2 \Delta^{2}+3 \sigma^{2}\right)\left(\Delta^{2}-6 \sigma^{2}\right) \operatorname{erfc}\left(\frac{\Delta}{\sqrt{2} \sigma}\right)+\left(21 \Delta^{2} \sigma^{2}+18 \sigma^{4}-2 \Delta^{4}\right) \operatorname{erfc}\left(\frac{\sqrt{2} \Delta}{\sigma}\right)\right)\right)\right]\right) .
\end{aligned}
$$

